\title{
Evidence for Processing Stages in Skill Acquisition: A Dual-Task Study
}

\author{
Udo Eversheim ${ }^{1}$ and Otmar Bock \\ Department of Physiology, German Sport University, D-50933 Cologne, Germany
}

\begin{abstract}
We investigated the changes of resource demand during the acquisition of a sensorimotor skill, namely the tracking of a visual target under reversed visual feedback. This acquisition task was performed alone or concurrently with one of four manual reaction-time tasks as loading tasks, designed to tap different computational resources. As expected, we found tracking performance to deteriorate upon vision reversal and then to gradually improve with practice. We further found that acquisition task and loading task interfered little before vision reversal but substantially afterwards. Most importantly, we observed a different time-course of interference for each of our four loading tasks. The particular pattern led us to conclude that resources related to spatial attention and sensory transformations are in highest demand early during skill acquisition and those pertinent to movement preparation somewhat later. Our findings thus provide experimental support for the theory that motor learning progresses in stages characterized by different resource requirements.
\end{abstract}

It has been suggested in the past that the acquisition of sensorimotor skills proceeds in distinct stages. For example, Fitts (1964) proposed a verbal-cognitive stage, in which subjects strive to comprehend task requirements and strategies; a motor stage, in which response patterns are gradually formed with the help of sensory feedback; and an autonomous stage, in which those patterns are integrated into larger sequences that can be run off with little demand on attention. Other investigators have presented similar stage models, with slight variations in the number and characteristics of stages (Pew 1966; Adams 1971; Shiffrin and Schneider 1977; Anderson 1982). Some of these differences could be due to the fact that the models pertained to different types of skills.

One approach to investigate the existence and nature of stages in skill acquisition is the dual-task paradigm. An acquisition task (AT) is combined with a concurrent loading task (LT). When the two tasks interfere, that is, when performance scores of AT and/or LT under dual-task conditions are lower than under single-task conditions, it is assumed that both tasks compete for the same class of informationprocessing resources in the brain. This interpretation follows the view that processing resources are limited and shareable (e.g., Kahnemann 1973; Navon and Gopher 1979) and that they can be subdivided into several classes (Wickens 1991). If skills indeed develop in stages, we should expect that the different processing demands of different stages are reflected by maximum interference with different

${ }^{1}$ Corresponding author.

E-MAIL eversheim@hrz.dshs-koeln.de; FAX 49-221-4982679. Article and publication are at http://www.learnmem.org/cgi/doi/ $10.1101 / \mathrm{lm} .39301$. loading tasks. It has therefore been postulated that AT will interfere with some LT early in training and with others later on while the overall magnitude of interference will gradually decay (Heuer 1984; Lintern and Wickens 1991).

A number of experimental studies have applied the dual-task paradigm to scrutinize the acquisition of various motor skills, thereby employing different combinations of AT and LT. Two studies (Bahrick et al. 1954; Trumbo et al. 1968) measured the effect of a concurrent LT on AT performance only after completion of learning, which makes meaningful interpretations very difficult. In the other studies, effects of LT on AT was determined early and late in training (Mohnkopf 1933; Noble et al. 1967; McLeod 1973; Redding and Wallace 1985; Redding et al. 1985; Nissen and Bullemer 1987; Kramer and Strayer 1988; Hazeltine et al. 1997; Ingram et al. 2000). AT performance was generally poorer under dual-task conditions but improved with training even if LT was administered continuously throughout the acquisition period; this finding was interpreted as evidence that dual-task interference does not prevent the acquisition of new skills. In some of these studies, AT improved by a similar amount with and without LT (Noble et al. 1967; McLeod 1973; Kramer and Strayer 1988; Ingram et al. 2000); whereas others found less improvement with LT (Redding and Wallace 1985; Redding et al. 1985). The remaining work provides no conclusive statistical evidence regarding this point, but the published data exhibit trends for less (Hazeltine et al. 1997), or more (Mohnkopf 1933; Nissen and Bullemer 1987) improvement in the presence of LT.

Unfortunately, an unequivocal interpretation of the above data is hampered by several methodological shortcomings. First, some studies employed separate subject

LEARNING \& MEMORY 8:183-189 @ 2001 by Cold Spring Harbor Laboratory Press ISSN1072-0502/01 \$5.00

$$
\begin{array}{lllllllllllllll}
\text { L } & E & A & R & N & I & N & G & \mathcal{Z} & M & E & M & O & R & Y \\
\text { ww.learnmem.org } & & &
\end{array}
$$


groups for single- versus dual-task conditions, such that intersubject variability could have blurred the effect of loading. Other studies tested the same subjects once with and once without LT, such that serial-order effects could be confounded with the effect of loading. Only a few studies employed an interleaved design (alternating episodes of single- and dual-task testing), which appears to be the optimal approach to minimize such problems.

Second, most studies measured AT but not LT performance. However, interference can manifest in AT, LT, or both, depending on the priority assigned by the subjects to each task (Wickens 1991). If registration is limited to AT scores, strategic shifts of task priority can be misjudged as changes in the magnitude of interference.

Third, most studies provided no control for causes of dual-task interference other than resource competition. Interference could result from costs of concurrence (Navon and Gopher 1979), that is, the effort of time-sharing per se or from a competition for control of peripheral inputs and/ or outputs (e.g., Klapp et al. 1987). Compelling evidence for a role of resource competition was provided only by one study, which used two levels of LT difficulty (Kramer and Strayer 1988), and two studies that included a nonlearning control for AT (Trumbo et al. 1968; Hazeltine et al. 1997): Either manipulation should have no effect on time sharing or on peripheral competition; however, both altered the magnitude of dual-task interference, thus supporting the role of resource competition.

Besides their methodological shortcomings, the experimental design of the cited studies is not suitable to scrutinize the existence of stages. Rather, the AT should be paired with several LT, each likely to require a different class of resources, and interference should be measured at multiple times during acquisition. Only a few of the cited studies measured interference at multiple times, and only one study employed two different LT.

The only available study using a battery of loading tasks (Logie et al. 1989) dealt with the acquisition of a complex computer game. This AT was combined with six different LT after 3 $\mathrm{h}$ and again after $8 \mathrm{~h}$ of training. Early in practice, the overall game score was significantly degraded by visuo-spatial and verbal LT but only marginally by a tapping LT. The degradation grew stronger with extended practice and became significant for tapping as well. Scores of individual game components (e.g., the number of times that one's spaceship was damaged by the enemy) exhibited an intricate pattern of component $\mathrm{x}$ LT interference; the magnitude of interference increased with practice and more components were affected. Unfortureversal. nately, the observed interference pattern is difficult to interpret since each component still comprises a variety of skills.

Logie et al. (1989) interpreted their findings as evidence that the demand for processing resources increased during practice on their AT and that some resource classes came into play only later in training. Due to the complex nature of the game, only the earliest stages of skill acquisition might have been captured and resource demand might well have dropped again with more extensive training.

Our present study employs a similar design as Logie et al. (1989), with some modifications to facilitate data interpretation. First, our AT is relatively simple; it probably does not involve multiple skills, it can be compactly represented by a single measure, and it is learned in limited time without excessively fatiguing the subjects. Second, we present our LT battery repeatedly during practice to sample the acquisition process more often. Third, we control for interference not related to resource competition in learning by including a nonlearning control.

\section{RESULTS}

Typical examples of tracking performance are shown in Figure 1 for four episodes of one subject. It can be seen that tracking accuracy was reasonably good under normal visual feedback, both in single- and dual-task conditions but deteriorated following up-down reversal, particularly in the dual-task situation. From data such as those in Figure 1, we calculated root mean square tracking error (RMSE) sepa-
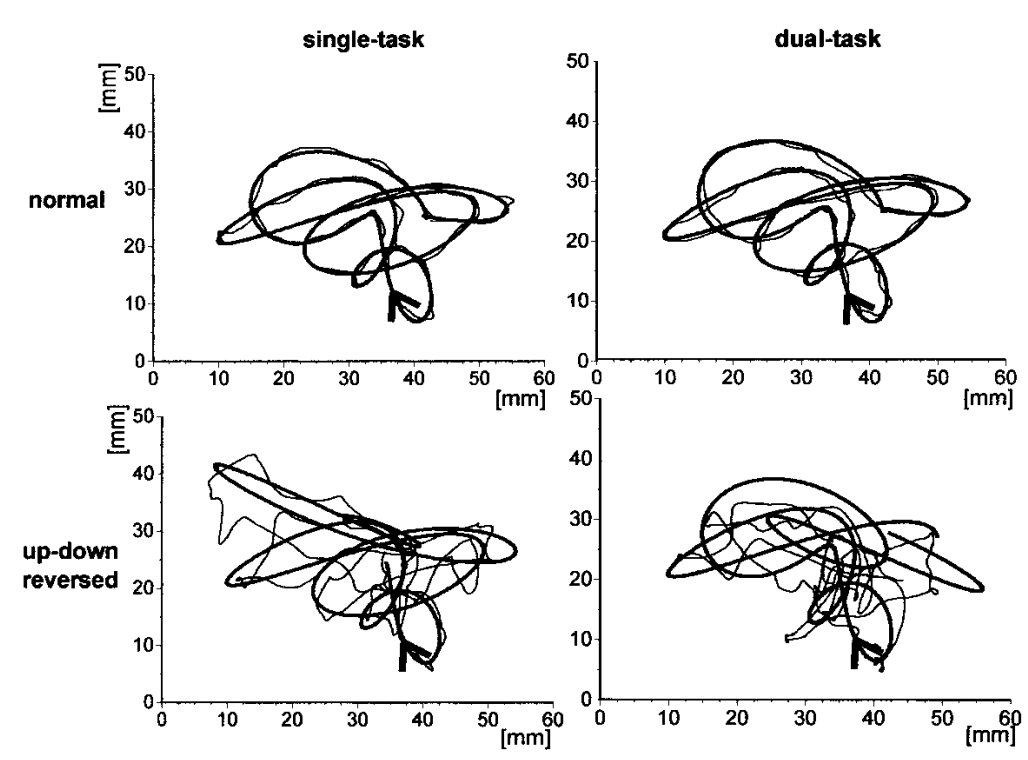

Figure 1 Original data of target (bold line) and cursor (thin line) paths in four experimental episodes of one subject under normal viewing conditions (top graphs) and immediately after up-down reversal (bottom graphs). Responses in single- (left graphs) and dual-task (right graphs) conditions are shown. Target movement direction is indicated by an arrow. Note the deterioration of tracking performance after

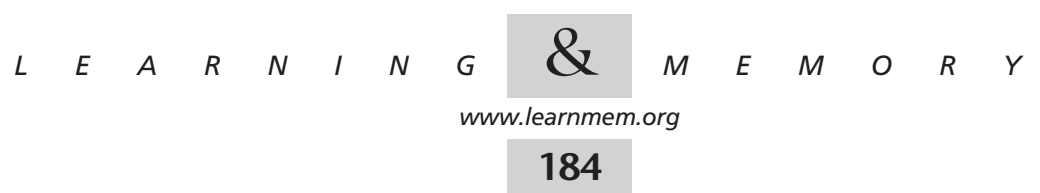


rately for each subject and episode. The outcome across subjects is summarised in Figure 2. It can be seen that before vision reversal, single- and dual-task RMSE scores were similar. This was confirmed by t-tests, yielding no significant differences between single task and either of the four dual tasks (all $P>0.05$ ).

Further from Figure 2, single-task RMSE increased abruptly upon vision reversal and then gradually decayed towards the baseline without reaching it completely. A discontinuity can be discerned after episode 31 , that is, following the rest break. We did not anticipate this phenomenon and attribute it to factors such as consolidation in motor memory (e.g., Brashers-Krug et al. 1996) or recovery from fatigue. Dual-task RMSE increased even more dramatically upon reversal and recovered with a roughly similar time course, such that a substantial difference between both data sets persisted throughout training.

We quantified the difference between single- and dualtask performance ( $\triangle$ RMSE) by the following procedure. As a first step, the single-task data of each subject were fitted by a one-phase exponential function up to episode 31 and by a regression line thereafter. The curve in Figure 2 shows the outcome of such a fit when applied to the averaged data. As a second step, $\triangle$ RMSE was calculated as the difference between each dual-task score and the pertinent fitted single-task score (Thus, a dual-task score achieved by a given subject in episode $j$ was contrasted with that subject's single-task score predicted for the very same episode $j$ ). In this way, effects of LT type were not confounded with those of serial order. The outcome of this procedure is illustrated in Figure 3, separately for each of the four LT-versions.

From Figure 3, $\triangle$ RMSE was negligible under normal

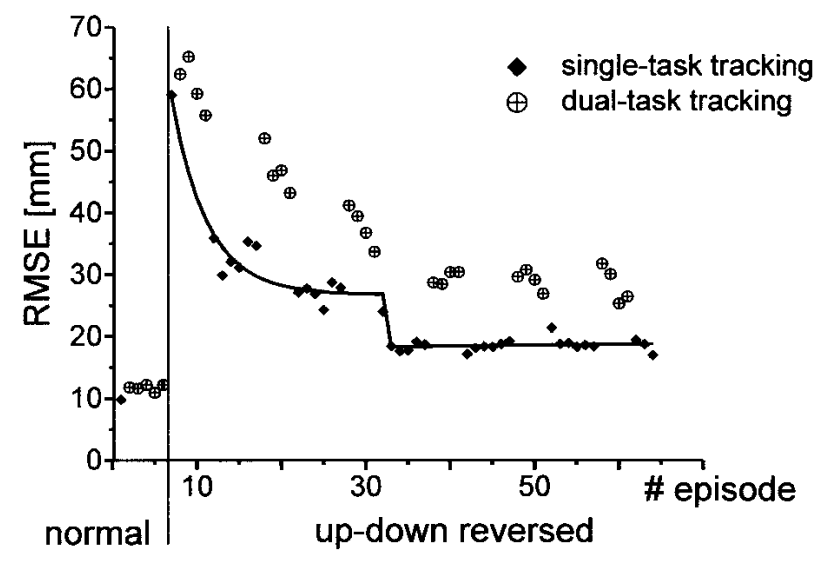

Figure 2 Time course of RMSE for all 64 episodes of the experiment. Each data point represents the mean RMSE of 12 subjects. Diamonds denote single-task tracking and circles dual-task tracking. The dashed vertical line indicates the onset of vision reversal. The bold curve represents an exponential (episodes 7-32) and a linear (episodes 33-64) fit to the single-task RMSE data. Dual-task data are plotted in the order in which they were administered to the individual subject and are not rearranged by loading task type.

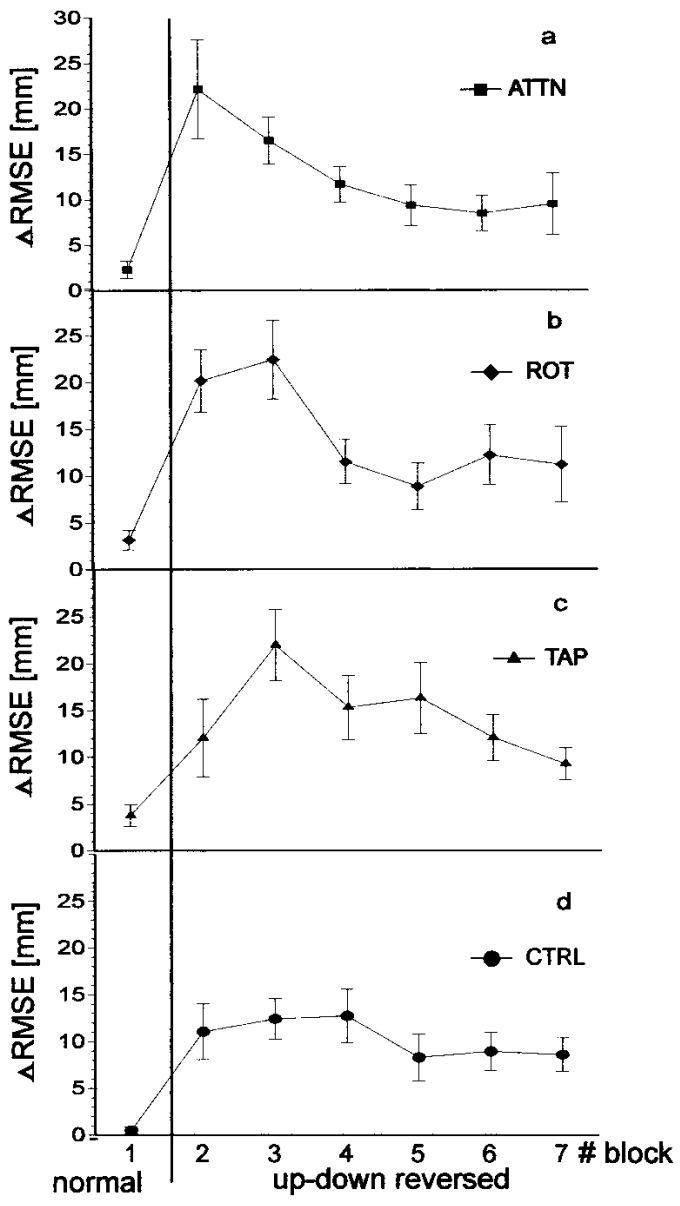

Figure 3 Dual-task performance for the tracking task is shown as mean dual-task decrement $\triangle$ RMSE (the difference between each subject's dual-task score and his/her expected single-task score for the same episode) and its standard error across subjects. After the first dual-task block, visual feedback of the tracking task was updown reversed. The values are sorted according to the concurrently performed loading task: ATTN (attention), ROT (rotation), TAP (tapping), and CTRL (control). Note that the time course of $\triangle$ RMSE differs between loading tasks.

vision, increased distinctly upon reversal, and did not return to the baseline by the end of training; these observations were expected from the data in Figure 2. However, $\Delta$ RMSE scores did not remain constant throughout training. Rather, they changed with time and-most importantly-followed a different time course for different LT-versions.

A two-way repeated-measures ANOVA $(7$ Blocks $\times 4$ LT) revealed a significant effect of Block $\left(\mathrm{F}_{(6.66)}=12.73\right.$; $P<0.001)$. Fisher's LSD tests yielded that Block 1 differed from all subsequent blocks (all $P<0.001$ ), Blocks 2 and 3 differed from Blocks 5-7 (all $P<0.01$ ), and Blocks 5-7 did not differ among themselves (all $P>0.05$ ). We therefore conclude that overall $\triangle$ RMSE increased after reversal and dropped later on without reaching the baseline value. The ANOVA further revealed significant effects of LT

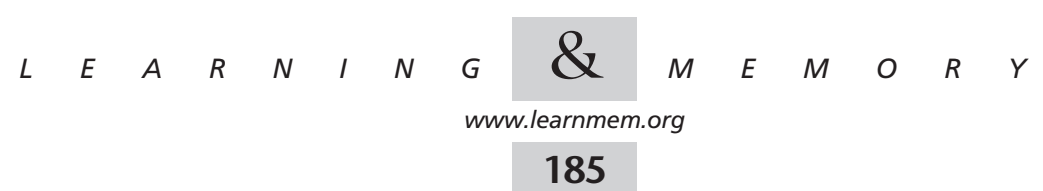


$\left(\mathrm{F}_{(3.33)}=3.64 ; P<0.05\right)$ and Block $\mathrm{x}$ LT $\left(\mathrm{F}_{(18.198)}=1.92\right.$; $P<0.05$ ). The latter finding is of particular interest, confirming that the time course of $\triangle$ RMSE was LT-specific. To explore this result, we compared CTRL to the other LT versions by Fisher's LSD tests. We found that the scores did not differ between tasks in Block 1 (all $P>0.05$ ). However, ATTN (attention) yielded significantly higher scores in Block $2(P<0.001)$, ROT (rotation) in Blocks 2 and 3 (both $P<0.001$ ), and TAP (tapping) in Blocks 3 and 5 (both $P<0.01$ ). We conclude from these analyses that following reversal, $\triangle$ RMSE increased with an early peak for ATTN, an early prolonged peak for ROT, and a still later peak for TAP.

Subjects' performance on the loading tasks is summarised in Figure 4. It can be seen that RT (reaction time) increased upon reversal and then somewhat dropped; whereas the differences between LT versions were maintained before and throughout training. These observations were confirmed by statistical analyses. A repeated-measures ANOVA $(7$ Blocks $\times 4$ LT) yielded a significant effect of Block $\left(\mathrm{F}_{(6.66)}=16.66 ; P<0.001\right)$; Fisher's LSD tests confirmed that RT in Block 1 was smaller than in the remaining blocks, in Block 2 higher than in the subsequent blocks (all $P<0.001$ ), and that RT did not differ between Blocks 5-7 (all $P>0.05$ ). We also found a significant effect of loading task $\left(\mathrm{F}_{(3.33)}=48.77 ; P<0.001\right)$, with ATTN yielding lower scores and ROT yielding higher scores than CTRL and TAP (all $P<0.001$ ). As expected, no significant interaction was found between Blocks and LTs $\left(\mathrm{F}_{(18.198)}=0.82 ; P>0.05\right)$.

\section{DISCUSSION}

Our study investigated the changes of resource demand during acquisition of a sensorimotor skill. We combined an acquisition task (AT, adaptation to vision reversal) with four different loading tasks (LT, four-choice RT task), designed to tap different resource classes. An interleaved design, with alternating episodes of single- and dual-task testing, was used to minimize the confounding effects of intersubject variability and serial order (see above).

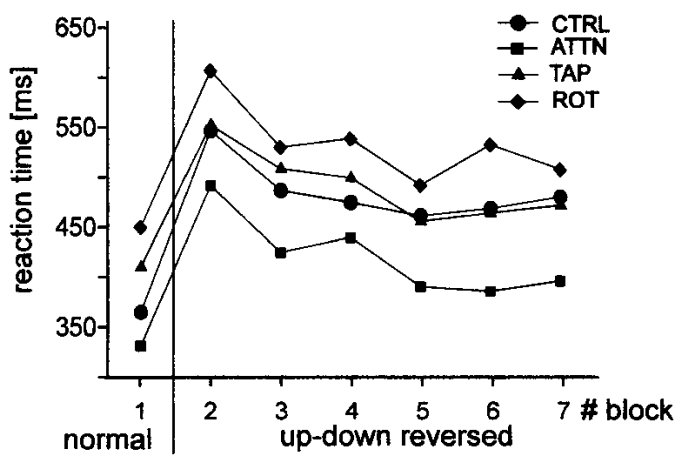

Figure 4 Dual-task performance across subjects for the reactiontime tasks under both normal and up-down reversed visual feedback of the tracking task. The reaction-time data are sorted by performed task type: CTRL (control), ATTN (attention), ROT (rotation), and TAP (tapping).
We will first consider the dual-task data from condition CTRL. If compared to the preadaptation baseline, our subjects' performance degraded markedly at the onset of reversal and recovered only in part later on. This degradation cannot be interpreted as a shift of task priority since it was found both in $\triangle$ RMSE and RT; it rather seems to reflect an increase of dual-task interference. The most likely reason for such an increase is an elevated demand for computational resources since other causes of interference (cost of timesharing per se and competition for peripheral inputs and outputs; see above) should be similar before and during skill acquisition. We therefore conclude that our adaptation task led to a long-lasting increase of resource demand. It remains open, however, exactly which resources were in high demand: Choice RT tasks such as CTRL require remarkably complex information processing (e.g., Sanders 1980) and may therefore utilize more than a single resource class.

More specific conclusions about the involved resources can be obtained by comparing CTRL to the other LT versions. Thus, the RT's results from ATTN indicate that subjects' ability to focus their attention, and thus to reduce RT, was not altered during adaptation. However, the sustained benefit of ATTN came at a price: $\triangle$ RMSE increased in ATTN substantially more than in CTRL during the first block under vision reversal, that is, dual-task interference during that block was higher in ATTN than in CTRL. These findings support the view that resources related to ATTN are in particularly high demand during early phases of sensorimotor adaptation.

Following the same argument, our findings indicate that during adaptation, dual-task interference reaches its maximum in ROT somewhat more prolonged than in ATTN and still later in TAP. This pattern seems to indicate that resources related to attention are in highest demand early during adaptation, those related to spatial transformations somewhat later, and those pertinent to movement preparation still later. Furthermore, the convergence of all $\triangle \mathrm{RMSE}$ scores to a similar above-baseline value during the final blocks suggests that later phases of training require resources that are utilized equally by all our LT.

It is important to note that performance data, such as the present RMSE scores, do not necessarily reflect the progress of learning; indeed, previous work has documented the existence of a possible dissociation between performance and learning scores (e.g., Shea and Morgan 1979; Willingham et al. 1997). Thus, the tracking decrement $\triangle$ RMSE in Figure 4 certainly reflects an effect of secondary tasks on performance but not necessarily an effect on learning; experimental designs other than the present one would be suitable to determine an interference with learning processes (e.g., Redding and Wallace 1985). Nevertheless, even if our secondary tasks did not slow down learning, their differential effects on tracking performance suggest that the resources needed to carry out the move-

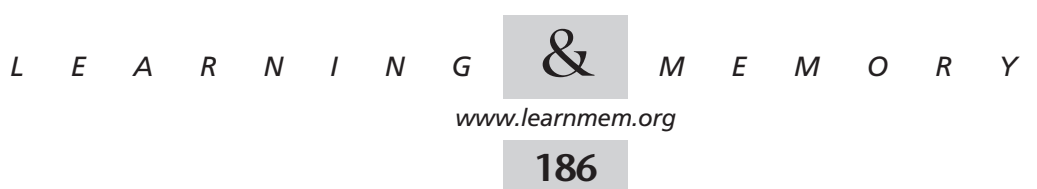



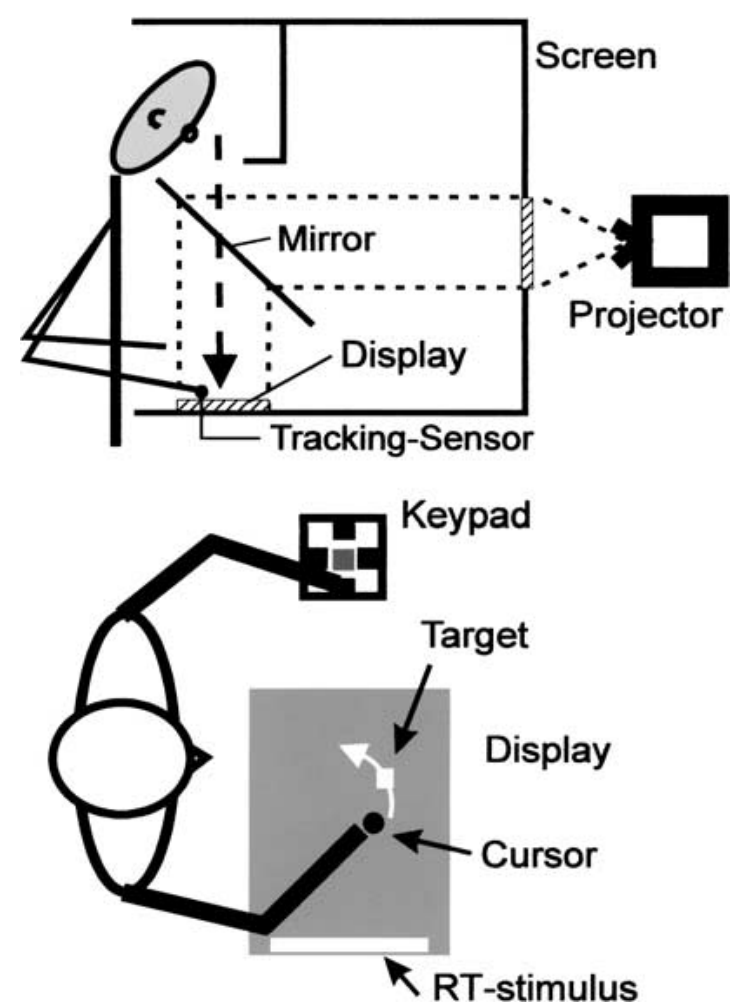

Figure 5 Schematic representations of the experimental setup in side and top view. A visual display (shaded) was presented through a tilted mirror, such that its virtual image appeared in a horizontal plane. A moving visual target was presented, and subjects tracked it with the index finger of their dominant arm; index finger position was registered by a sensor and presented in the display as cursor position. Peripheral light bars (reaction time [RT]-stimulus) were also displayed and subjects responded by depressing the corresponding key on a keypad, using their nontracking arm. The mirror precluded vision of both arms.

ment will change qualitatively and quantitatively during adaptation. This finding is in accordance with the view that during skill acquisition, the sensorimotor system passes through phases characterized by different resource requirements (cf. Heuer 1984; Lintern and Wickens 1991). In particular, sensorimotor adaptation in the present study seems to be acquired in a stage in which attention-demanding processing is particularly important and in which spatial relationships are adjusted, followed by a stage where the formulation of new response patterns predominates.

Available models of skill acquisition (see above) agree well with the specific stage structure suggested by the present data. Thus, Fitts' (1954) verbal-cognitive stage involves substantial mental reasoning and may require resources related to mental rotation and could well be attention-demanding; the subsequent motor stage may rely on resources related to the improvement of adequate movement patterns; the final autonomous stage is characterized by reduced attentional requirements and increasing automatic processing.
Any stages subsequent to the ones mentioned above are difficult to determine with the present data since the interference pattern of all our LT coincided late in training. Additional experiments, using a new battery of LT, would be needed to elucidate this point. It would also be desirable to find out in future experiments if the interference pattern observed in the present study is specific for the AT used or rather generalizes across a range of diverse ATs.

\section{MATERIALS AND METHODS}

Our experimental setup is outlined in Figure 5. A computer display presented on a back-projection screen was viewed through a tilted mirror, such that its virtual image $(60 \mathrm{~cm} \times 40 \mathrm{~cm})$ coincided with the surface of a tabletop in front of the subject. Because of the mirror, subjects were unable to see their arms.

Our AT was adaptation to up-down reversed visual feedback in a pursuit-tracking task. The tracking target was a white square of 2-cm side length, moving on the tabletop along an unpredictable trajectory. The position of the index fingertip of the dominant hand was registered by the Fastrak motion analysis system (based on electromagnetic induction), which has a spatial accuracy of $\sim 1 \mathrm{~mm}$ and a temporal resolution of $120 \mathrm{~Hz}$. The recorded signal was used to drive a red circular cursor on the display, and subjects were instructed to track the target by the cursor as accurately as possible. Tracking performance was quantified as a RMSE between cursor and target position. At the onset of each session, cursor position coincided with finger position, that is, subjects simply had to follow the target with their fingertip. Later on, cursor movement was up-down reversed, such that a finger movement toward the body yielded a cursor movement away from the body and vice versa. We have shown before that this manipulation produces initially a dramatic increase of RMSE, followed by a gradual recovery within about $30 \mathrm{~min}$ (Bock et al., in press). We have shown before that this manipulation produces initially a dramatic increase of RMSE, followed by a gradual recovery within about $30 \mathrm{~min}$ (Bock et al., in press).

The four different loading tasks were all four-choice manualRT tasks. Four possible stimulus positions were continuously presented as thin grey lines $(0.1 \mathrm{~cm} \times 36 \mathrm{~cm})$ along the four edges of the display. A trial started when the index finger of the subject's nondominant hand depressed the central key of a numeric keypad. After a randomized time interval of $700-1300 \mathrm{~ms}$, a wider, white bar $(1 \times 36 \mathrm{~cm})$ flashed for $100 \mathrm{~ms}$ in one of the four positions (see Fig. 5), as determined randomly in each trial. (Our rationale for flashing the target was to encourage subjects to watch the display periphery continuously, rather than shifting attention back and forth between LT and AT.) Subjects were instructed to depress the corresponding key on the keypad with their index finger as quickly as possible, and their RT was quantified as interval between stimulus appearance and release of the starting key. Responses to a false key, or with RT outside a window of 100-1500 ms, were excluded from further analysis ( $\sim 3 \%$ of all trials).

Four versions of the loading task were used. In CTRL, the task was exactly as described above, while each other version differed from CTRL in one respect. In ATTN (attention), a grey line appeared only along the edge where the next stimulus would appear and thus served as a precue about stimulus position. Such precues are known to reduce RT. They do not act by facilitating the preparation of movements since precues remain effective even when they do not specify any particular response parameter (e.g., Good-

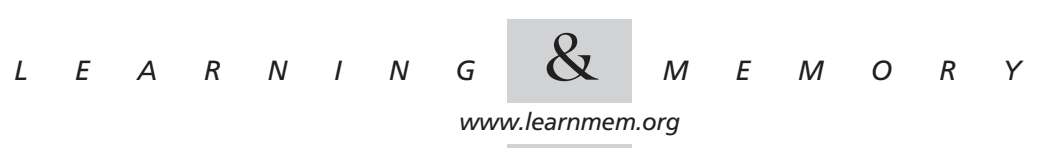


man and Kelso 1980; Bock and Eversheim 2000). Rather, they probably act by focusing subjects' attention within the area of space where the target is going to appear (e.g., Posner et al. 1980; Murphy and Eriksen 1987; U. Eversheim and O. Bock, unpubl.). In TAP, the target key had to be depressed in a short-long-short sequence, that is, a more complex response was required. It is known that RT increases with response difficulty, probably due to the higher complexity of preparatory processing (Henry and Rogers 1960). In ROT, responses were rotated $90^{\circ}$ counter-clockwise with respect to the stimuli, that is, subjects had to depress the right key when the bar flashed at the bottom. The increase of RT under such conditions has been attributed to the computational costs of spatial transformations (Georgopoulos and Massey 1987). We expected that an increased demand for resources related to attention, movement preparation, or spatial transformations should yield an increased interference in ATTN, TAP, or ROT, respectively, if compared to the interference in CTRL. In consequence, changing resource demands during skill acquisition should yield a changing interference pattern.

Each task was executed in episodes of 35-sec duration, separated by breaks of self-determined length, mostly 1-2 sec. The four LT versions were administered as a block of four consecutive episodes, and their order within each block was counterbalanced across subjects and blocks. Each subject participated on two separate days, one day to familiarize with our tasks and one to collect data.

Day 1 consisted of five blocks of LT (i.e., $5 \times 4=20$ episodes of 35-sec duration) under single-task conditions, followed by one episode of AT and then by five blocks of LT and AT under dual-task conditions. The relationship between finger and cursor movement in AT was not reversed on this day. We observed that single- and dual-task performance reached a plateau during testing, and concluded that our subjects were sufficiently familiarized with their tasks.

Day 2 was scheduled 1-7 d later than $\mathrm{d} 1$. It started again with normal visual feedback about the tracking finger. Subjects completed one episode of AT alone, one episode under dual-task conditions (which served as warmup and was not analyzed), and then one block of dual-task episodes. Visual feedback was then reversed and AT was administered under single- and dual-task conditions as per the schedule at the bottom of Figure 2. Taken together, d 2 consisted of 64 episodes, or nearly $40 \mathrm{~min}$ of performance. To avoid fatigue and loss of motivation, a five-min break was introduced after episode 31 .

Twelve healthy humans, aged 21-30 y, participated in our study. Three were male and nine female, and ten were righthanded. None of them exhibited overt sensorimotor deficits besides corrected vision, and none had participated before in studies on skill acquisition; this is important, because successive training sessions interact even after months without practice (Bock et al., in press). All subjects gave their informed consent to this study, which was pre-approved by the local ethics committee.

\section{ACKNOWLEDGMENTS}

The present study was supported by the German Ministry for Education and Research (DLR grant 50 WB 9942); responsibility for the contents rests with the authors. We thank D. Papajewski for his help with data collection and analysis. Parts of the present work were presented in abstract form at the 2000 annual meeting of the Society of Neuroscience.

The publication costs of this article were defrayed in part by payment of page charges. This article must therefore be hereby marked "advertisement" in accordance with 18 USC section 1734 solely to indicate this fact.

\section{REFERENCES}

Adams, J.A. 1971. A closed-loop theory of motor learning. J. Mot. Behav. 3: 111-149.

Anderson, J.R. 1982. Acquisition of cognitive skill. Psychol. Rev. 89: 369-406.

Bahrick, H.P., Noble, M.E., and Fitts, P.M. 1954. Extra-task performance as a measure of learning a primary task. J. Exp. Psychol. 48: 298-302.

Brashers-Krug, T., Shadmer, R., and Bizzi, E. (1996) Consolidation in human motor memory. Nature 382: 252-255.

Fitts, P.M. 1964. Perceptual-motor skill learning. In Categories of human learning (ed. A.W. Melton), pp. 243-285. Academic Press, NY

Georgopoulos, A.P. and Massey, J.T. 1987. Cognitive spatial-motor processes: 1 . The making of movements at various angles from a stimulus direction. Exp. Brain Res. 65: 361-370.

Hazeltine, E., Grafton, S., and Ivry, R. 1997. Attention and stimulus characteristics determine the locus of motor-sequence encoding. A PET study. Brain 120: 123-140.

Henry, F.M. and Rogers, D.E. 1960. Increased response latency for complicated movements and a "memory drum " theory of neuromotor reaction. Res. Q. 31: 448-458.

Heuer, H. 1984. Motor learning as a process of structural constriction and displacement. In Cognition and motor processes (eds. W. Prinz and A.F. Sanders), pp. 295-305. Springer, Heidelberg.

Ingram, H.A., van Donkelaar, P., Cole, J., Vercher, J.-L., Gauthier, G.M., and Miall, R.C. 2000. The role of proprioception and attention in a visuomotor adaptation task. Exp. Brain. Res. 132: 114-126.

Kahnemann, D. 1973. Attention and effort. Prentice-Hall, New Jersey.

Klapp, S.T., Kelly, P.A., and Netick, A. 1987. Hesitations in continuous tracking Iinduced by a concurrent discrete task. Hum. Factors 29: 327-337.

Kramer, A.F. and Strayer, D.L. 1988. Assessing the development of automatic processing: An application of dual-task and event-related brain potential methodologies. Biol. Psychol. 26: 231-267.

Lintern, G. and Wickens, C. 1991. Issues for acquisition and transfer of timesharing and dual-task skills. In Multiple task performance (ed. D. Damos), pp. 123-138. Taler \& Francis, Ltd., Bristol.

Logie, R., Baddelley, A., Mané, A., Donchin, E., and Sheptak, R. 1989. Working memory in the acquisition of complex cognitive skills. Acta Psychol. 71: 53-87.

McLeod, P.D. 1973. Interference of "attend to and learn" tasks with tracking. J. Exp. Psychol. 99: 330-333.

Mohnkopf, W. 1933 Zur Automatisierung willkürlicher Bewegungen. Z. Psychol. 130: 235-299.

Murphy, T.D. and Eriksen C.W. 1987. Temporal changes in the distribution of attention in the visual field in response to precues Percept. Psychophys. 42: 576-586.

Navon, D. and Gopher, D. 1979. On the economy of the human-processing system. Psychol. Rev. 86: 214-255.

Nissen, M.J. and Bullemer, P. 1987. Attentional requirements of learning: Evidence from performance measures. Cognit. Psychol. 19: 1-32.

Noble, M., Trumbo, D., and Fowler, F. 1967. Further evidence on secondary task interference in tracking. J. Exp. Psychol. 73: 146-149.

Pew, R.W. 1966. Acquisition of hierarchical control over the temporal organization of a skill. J. Exp. Psychol. 71: 764-771.

Posner, M.I. 1980 Orientating of attention. Q. J. Exp. Psychol. 32: 3-25

Redding, G.M. and Wallace, B. 1985. Cognitive interference in prism adaptation. Percept. Psychophys. 37: 225-230.

Redding, G.M., Clark, S.E., and Wallace, B. 1985. Attention and prism adaptation. Cognit. Psychol. 17: 1-25.

Sanders, A.F. 1980. Stage analysis of reaction processes. In Tutorials in Motor Behavior. (eds. G.E. Stelmach and J. Requin), pp. 331-354. North Holland, Amsterdam.

Shea, J.B. and Morgan, R.L. 1979. Contextual interference effects on the

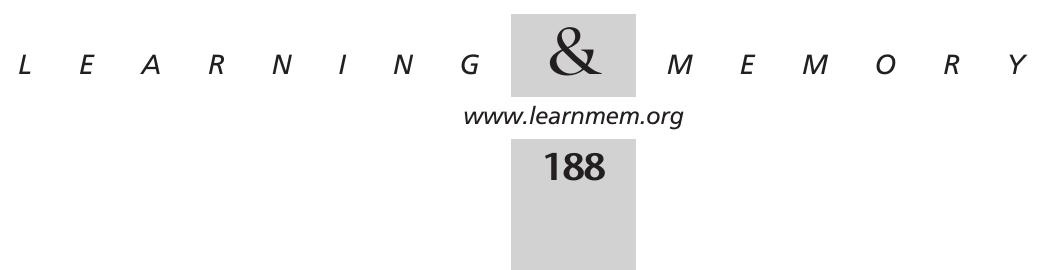


acquisition, retention, and transfer of a motor skill. J. Exp. Psychol. Hum. Learn. Mem. 5: 179-187.

Shiffrin, R.M. and Schneider, W. 1977. Controlled and automatic human information processing: II. Perceptual learning, automatic attending, and a general theory. Psychol. Rev. 84: 127-190.

Trumbo, D.A., Noble, M., and Quigley, J. 1968. Sequential probabilities and the performance of serial tasks. J. Exp. Psychol. 76: 364-372.

Wickens, C.D. 1991. Processing resources and attention. In Multiple Task
Performance (ed. D.L. Damos), pp. 3-34. Taler \& Francis, Ltd., Bristol. Willingham, D.B., Greenberg, A.R., and Thomas, R.C. 1997.

Response-to-stimulus interval does not affect implicit motor sequence

learning, but does affect performance. Mem. Cognit. 25: 534-542.

Received January 29, 2001; accepted in revised form June 8, 2001.

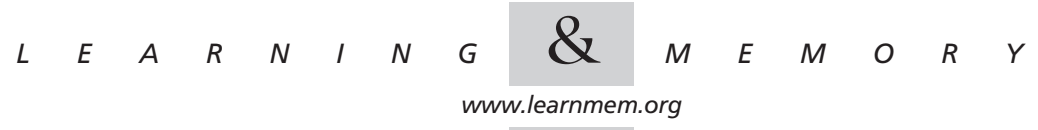




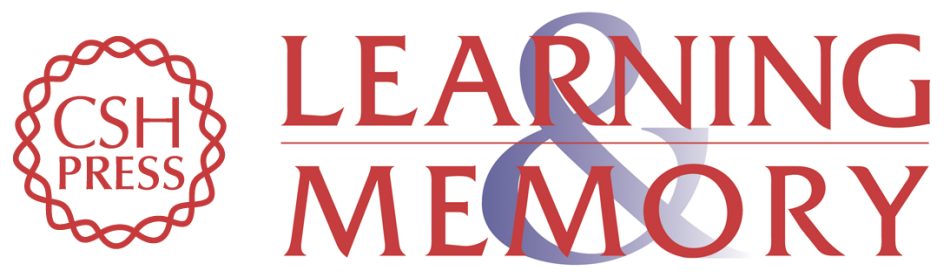

\section{Evidence for Processing Stages in Skill Acquisition: A Dual-Task Study}

Udo Eversheim and Otmar Bock

Learn. Mem. 2001, 8:

Access the most recent version at doi:10.1101//m.39301

\section{License}

Email Alerting

Service

Receive free email alerts when new articles cite this article - sign up in the box at the top right corner of the article or click here. 